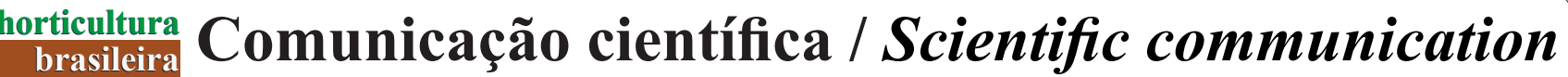

PAULA FLM; FRIZZONE JA; PAULAAL; MANFRON PA; SOARES TM; ROJAS JSD. 2015. Concentração foliar de nutrientes na pimenta 'Tabasco' em função da aplicação de CO2 via irrigação. Horticultura Brasileira 33: 224-229. DOI - http://dx.doi.org/10.1590/S0102-053620150000200014

\section{Concentração foliar de nutrientes na pimenta 'Tabasco' em função da aplicação de $\mathrm{CO}_{2}$ via irrigação}

\author{
Fabiana LM Paula'; Jose A Frizzone²; Adalberto L Paula ${ }^{3}$; Paulo A Manfron'; Tales M Soares; ;uan SD Rojas ${ }^{2}$ \\ ${ }^{1}$ UTFPR, R. Marechal Mascarenhas de Morais 91, São Francisco de Assis, 85660-000 Dois Vizinhos-PR; fabianadepaula3@hotmail. \\ com; ${ }^{2}$ USP-ESALQ, Depto. Eng. Rural, C. Postal 9, 13418-900 Piracicaba-SP; frizzone@usp.br; juansdrojas@hotmail.com; ${ }^{3}$ UTFPR, \\ Depto. de Agronomia, Estr. para Boa esperança km 04, 85660-000 Dois Vizinhos-PR; adalbertolpaula@utfpr.edu.br; ${ }^{4} U F S M$, Depto. \\ Fitotecnia, Av. Roraima, 1000, Camobi, 97105-900 Santa Maria-RS; pamanfron@hotmail.com; ${ }^{5}$ UFRB, Depto. Irrigação, R. Rui Barbosa \\ 710, 44380-000 Cruz das Almas-BA; tales@ufrb.edu.br
}

\section{RESUMO}

Foram avaliados os efeitos de diferentes doses de dióxido de carbono $\left(\mathrm{CO}_{2}\right)$, aplicadas via irrigação por gotejamento na concentração de macronutrientes e micronutrientes nas folhas da pimenta 'Tabasco' (Capsicum frutescens) em ambiente protegido. O delineamento experimental utilizado foi aleatorizado em blocos, com quatro tratamentos e oito repetições. Os tratamentos foram constituídos inicialmente de quatro doses de $\mathrm{CO}_{2}(0 ; 206,6 ; 309,9$ e $413,2 \mathrm{~kg} / \mathrm{ha})$ antes da poda das plantas, e posteriormente de mais quatro doses de $\mathrm{CO}_{2}(0 ; 245,4 ; 368,0$ e $490,7 \mathrm{~kg} / \mathrm{ha})$ após a poda, respectivamente para T1, T2, T3 e T4. A análise da concentração de nutrientes nas folhas foi realizada aos 189, 404 e 484 dias após o transplantio (DAT). Verificou-se efeito linear significativo $(\mathrm{p}<0,05)$, para as doses de $\mathrm{CO}_{2}$ sobre as concentrações de $\mathrm{N}, \mathrm{Fe}, \mathrm{Mn}$ e, para o Zn efeito quadrático significativo $(\mathrm{p}<0.05)$ aos 187 DAT. Com relação à concentração de $\mathrm{Ca}$, houve efeito linear decrescente significativo $(\mathrm{p}<0,01)$ aos 484 DAT. Observou-se que a concentração de $\mathrm{N}$ nas folhas aos 187 DAT aumentou em função das doses de $\mathrm{CO}_{2}$. Quanto ao $\mathrm{Fe}$, verificou-se que a concentração na folha diminuiu em função das doses de $\mathrm{CO}_{2}$. Esse mesmo comportamento foi observado em relação ao $\mathrm{Mn}$ aos 189 DAT, mas com um aumento em T4 em relação aos demais tratamentos. A concentração de $\mathrm{Zn}$ diminuiu nos tratamentos T2 e T3, com um aumento em $\mathrm{T} 4 \mathrm{em}$ relação à testemunha. $\mathrm{O} \mathrm{CO}_{2}$ teve efeito significativo nos teores de N, Fe, Mn e Zn aos 187 DAT e no Ca aos 484 DAT. O CO, ocasionou aumento na concentração de nitrogênio $(59.5 \mathrm{~g} / \mathrm{kg})$ e zinco $(97 \mathrm{mg} / \mathrm{kg})$ nas folhas de pimenta Tabasco comparado à testemunha com valores de $49,3 \mathrm{~g} / \mathrm{kg}$ e $86 \mathrm{mg} / \mathrm{kg}$ para valores de $\mathrm{N}$ e $\mathrm{Zn}$, respectivamente. A concentração de Fe $(230 \mathrm{mg} / \mathrm{kg}), \mathrm{Mn}$ $(235 \mathrm{mg} / \mathrm{kg})$ e Ca $(13,8 \mathrm{~g} / \mathrm{kg})$ teve um decréscimo nas folhas quando comparado à testemunha, com valores de $235 \mathrm{mg} / \mathrm{kg}, 308 \mathrm{mg} / \mathrm{kg}$ e $26,4 \mathrm{~g} / \mathrm{kg}$, para Fe, Mn e Ca, respectivamente.

Palavras chave: Capsicum frutescens, irrigação localizada, dióxido de carbono.

\begin{abstract}
Leaf nutrients concentration on 'Tabasco' pepper due to the application of $\mathrm{CO}_{2}$ by irrigation

We verified the effect of different doses of carbon dioxide $\left(\mathrm{CO}_{2}\right)$, supplied via drip irrigation in the concentration of macronutrients and micronutrients in the leaves of 'Tabasco' pepper (Capsicum frutescens). The treatments consisted initially of four levels of $\mathrm{CO}_{2}$ $(0,206.6,309.9$ and $413.2 \mathrm{~kg} / \mathrm{ha})$ before pruning the plants, and then four more doses of $\mathrm{CO}_{2}(0,245.4,368.0$ and $490.7 \mathrm{~kg} / \mathrm{ha})$ after pruning, respectively $\mathrm{T} 1, \mathrm{~T} 2, \mathrm{~T} 3$ and $\mathrm{T} 4$. The analysis of nutrient concentration was determined in leaves at 189, 350 and 432 days after transplanting (DAT). There was a significant linear effect $(\mathrm{p}<0.05)$ for rates of $\mathrm{CO}_{2}$ on concentrations of N, Fe, Mn and for $\mathrm{Zn}$ there was a quadratic effect $(\mathrm{p}<0.05)$ at 187 DAT. With respect to the concentration of $\mathrm{Ca}$, there was a significant linear effect $(\mathrm{p}<0.01)$ at 484 DAT. The $\mathrm{N}$ concentration in leaves at 187 DAT increased depending on the $\mathrm{CO}_{2}$ levels. The concentration of Fe decreased depending on the $\mathrm{CO}_{2}$ levels. Identical behavior was observed on $\mathrm{Mn}$ at $189 \mathrm{DAT}$. The concentration of $\mathrm{Zn}$ decreased on treatments T2 and T3, with an increase on T4 compared to control. We concluded that $\mathrm{CO}_{2}$ had a significant effect on the levels of $\mathrm{N}, \mathrm{Fe}, \mathrm{Mn}$ and $\mathrm{Zn}$ at 187 DAT and 484 DAT to $\mathrm{Ca}$. The $\mathrm{CO}_{2}$ caused an increase on the concentration of $\mathrm{N}(59.5 \mathrm{~g} / \mathrm{kg})$ and $\mathrm{Zn}(97 \mathrm{mg} / \mathrm{kg})$ on 'Tabasco' pepper leaves compared to the control values with $49.3 \mathrm{~g} / \mathrm{kg}$ and 86 $\mathrm{mg} / \mathrm{kg}$ for values of $\mathrm{N}$ and $\mathrm{Zn}$, respectively. The concentration of $\mathrm{Fe}$ $(230 \mathrm{mg} / \mathrm{kg}) \mathrm{Mn}(235 \mathrm{mg} / \mathrm{kg})$ and $\mathrm{Ca}(13.8 \mathrm{~g} / \mathrm{kg})$ decreased in the leaves as compared to control, with values of $235 \mathrm{mg} / \mathrm{kg}, 308 \mathrm{mg} / \mathrm{kg}$ and $26.4 \mathrm{~g} / \mathrm{kg}$ for $\mathrm{Fe}, \mathrm{Mn}$ and $\mathrm{Ca}$, respectively.
\end{abstract}

Keywords: Capsicum frutescens, trickle irrigation, carbon dioxide.

(Recebido para publicação em 15 de outubro de 2013; aceito em 15 de novembro de 2014) (Received on October 15, 2013; accepted on November 15, 2014)

\begin{abstract}
A s pimentas constituem importante segmento do setor de hortaliças, tanto para agricultura quanto para a indústria alimentícia. Do ponto de vista
\end{abstract}

social, o cultivo da pimenta tem grande importância devido à exigência de mão de obra, principalmente na colheita (EPAMIG, 2006). Aárea anual cultivada no Brasil é de dois mil hectares, e os principais estados produtores são Minas Gerais, Goiás, São Paulo, Ceará e Rio Grande do Sul. No Ceará, a pimenta 
‘Tabasco' é exportada na forma de pasta, para ser utilizada na fabricação do molho Tabasco (EPAMIG, 2006).

A cultura da pimenta 'Tabasco' requer temperatura média do ar entre 25 a $35^{\circ} \mathrm{C}$ (Khan et al., 2005). Trata-se de uma cultura de clima tropical, exigente em calor, sensível à baixa temperatura e intolerante a geadas; por esses motivos deve ser cultivada nas épocas mais quentes do ano. As condições encontradas na região de Piracicaba-SP proporcionam o cultivo satisfatório desse tipo de pimenta, que possui produtividade média variando de 10 a 30 t/ha (Embrapa, 2006). Com investimento em tecnologias como a aplicação de $\mathrm{CO}_{2}$ via água da irrigação é possível aumentar a produtividade da pimenta Tabasco em 16\% (Paula et al., 2011).

A análise foliar juntamente com a análise do solo, é utilizada para avaliar o estado nutricional das culturas para estabelecer as recomendações de adubação, identificando quais nutrientes são limitantes para a produção. $\mathrm{O}$ teor do nutriente na planta é resultante da ação e interação dos fatores que afetam a sua disponibilidade no solo e sua absorção pelas plantas (Portz et al., 2006). Os minerais têm papel importante do ponto de vista nutricional para a planta, sendo exigidos em maior ou menor concentração, cada um com uma finalidade especifica nas plantas (Bezerra Neto et al., 2008).

A aplicação de $\mathrm{CO}_{2}$ via água de irrigação vem sendo utilizada com o intuito de melhorar a absorção de nutrientes pelas culturas, em função do efeito de abaixamento temporário do $\mathrm{pH}$ do solo. $\mathrm{O} \mathrm{CO}_{2}$ provoca redução do $\mathrm{pH}$ do solo, aumentando a disponibilidade de alguns nutrientes presentes no solo. O efeito de redução do $\mathrm{pH}$ pode estar associado à ocorrência de bicarbonatos de cálcio, provenientes da reação de ácidos carbônicos formados pela aplicação de $\mathrm{CO}_{2}$ via água de irrigação com o Ca presente no solo (Pinto et al., 2000).

A variação da acidez do solo, até níveis adequados para a vida das plantas, em torno de 5,5 a 6,3 (Malavolta, 2006), pode aumentar ou diminuir a disponibilidade de macronutrientes e de micronutrientes no solo para as plantas, fato este que está associado à resposta das plantas na absorção de nutrientes em solos com a adição de agua carbonatada via irrigação, pela diminuição momentânea do pH do solo (Pinto et al., 2000).

A demanda por alimentos de melhor qualidade requer o uso de tecnologias que minimizem os efeitos que limitam a produtividade. Dentre estas tecnologias, tem-se o uso do dióxido de carbono $\left(\mathrm{CO}_{2}\right)$ aplicado via irrigação, com o objetivo de maximizar a produtividade, melhorar a qualidade de frutos e reduzir os custos de produção (Pinto et al., 2001; Cararo \& Duarte, 2002; Furlan et al., 2002; Frizzone et al., 2005a, 2005b; D'Alburquerque Junior et al., 2007; Araújo \& Botrel, 2010; Paula et al., 2011).

A falta de informação sobre o efeito da aplicação de $\mathrm{CO}_{2}$ na cultura da pimenta tem limitado a utilização desta técnica, sendo necessários estudos para determinar as doses de $\mathrm{CO}_{2}$ mais adequadas para otimizar seu cultivo, em ambiente protegido. Este trabalho teve como objetivo analisar os efeitos de doses de dióxido de carbono, aplicadas via irrigação por gotejamento, sobre a concentração de macro e micronutrientes nas folhas da pimenta 'Tabasco' (Capsicum frutescens).

\section{MATERIAL E MÉTODOS}

$\mathrm{O}$ experimento foi conduzido em ambiente protegido, instalado em área experimental da Escola Superior de Agricultura "Luiz de Queiroz" (ESALQ), localizada no município de Piracicaba-SP $\left(22^{\circ} 42\right.$ '30"S, 47³8'00'”, $580 \mathrm{~m}$ de altitude).

O solo foi classificado como Latossolo Vermelho-Amarelo Álico. As análises químicas (Tabela 1) foram realizadas no Laboratório de Solos e Nutrição de Plantas segundo Novais et al. (2007) e as análises físicas segundo Camargo et al. (2009), realizadas no Laboratório de Água e Solo do Departamento de Engenharia Rural da ESALQ. As análises físicas revelaram: argila (\%), silte $(\%)$ e areia $(\%)$ de $15,23,8,67$ e 76,10 , respectivamente e massa especifica de solo e partículas de 1,08 e 2,66 g/ $\mathrm{cm}^{3}$, respectivamente.

$\mathrm{O}$ experimento foi conduzido em duas casas de vegetação do tipo arco em aço galvanizado, com orientação leste-oeste, com 3,0 $\mathrm{m}$ de pé direito, 7,0 $\mathrm{m}$ de largura, 17,5 $\mathrm{m}$ de comprimento e 4,7 m de altura, cobertas com polietileno transparente com $150 \mu$ de espessura, com tratamento anti UV e com abertura sob cada arco das fachadas frontais para circulação do ar.

O delineamento experimental foi em blocos casualizados, com quatro repetições por casa de vegetação. Os tratamentos aplicados foram constituídos inicialmente de quatro doses de $\mathrm{CO}_{2}(0 ; 206,6 ; 309,9$ e 413,2 kg/ ha) antes da poda das plantas, e posteriormente de mais quatro doses de $\mathrm{CO}_{2}$ $(0 ; 245,4 ; 368,0$ e 490,7 kg/ha) após a poda, respectivamente $\mathrm{T} 1, \mathrm{~T} 2, \mathrm{~T} 3 \mathrm{e}$ T4. Estas doses foram definidas com base em trabalhos prévios, conduzidos com aplicação de $\mathrm{CO}_{2}$ nas culturas de melão (Pinto et al., 2001; Frizzone et al., 2005a, D'Albuquerque Junior et al., 2007). As doses de $\mathrm{CO}_{2}$ foram parceladas em 70 aplicações, sendo 32 antes da poda e 38 após a poda, com duração do ciclo de desenvolvimento da cultura de 1 ano e 4 meses, resultando nas seguintes doses por aplicação durante todo o ciclo: 6,46; 9,68 e 12,91 kg/ha.

O monitoramento dos elementos microclimáticos foi realizado mediante auxílio de um "datalogger", que forneceu as médias de leituras de sensores a cada 30 minutos. Os sensores instalados foram dois psicrômetros com termopares de bulbo úmido e seco (temperatura e umidade relativa), sendo um por casa-de-vegetação, além de um saldo-radiômetro (radiação líquida). Os sensores foram instalados no centro da casa de vegetação.

O espaçamento de transplantio utilizado foi de $80 \mathrm{~cm}$ entre plantas por 1,10 m entre linhas com uma população 11.363 plantas/ha. Cada casa-de-vegetação (estufas) apresentava quatro blocos. Cada bloco apresentava seis linhas de plantas de 4,0 $\mathrm{m}$ de comprimento, totalizando 30 plantas por bloco, sendo as duas linhas laterais as bordaduras. A área útil do bloco foi constituída de 12 plantas, utilizadas para as avaliações realizadas durante o período experimental.

A semeadura foi realizada em 
08/08/06, e o transplantio aos 50 dias após a semeadura (DAS), quando as mudas apresentaram seis folhas definitivas. Com base na análise química e na recomendação de adubação para o estado de São Paulo (Raij et al., 1997), 30 dias antes do transplantio, incorporou-se NPK nas doses de 40, 600 e $180 \mathrm{~kg} / \mathrm{ha}$ na forma de uréia, superfosfato simples e cloreto de potássio, respectivamente.

Foram realizadas duas podas apicais, a primeira 11 dias após o transplantio (10/10/06), com o objetivo de induzir a planta a emitir brotações laterais. A segunda foi realizada 16 dias após a primeira (26/10/06), tendo como finalidade aumentar o número de galhos e deixar a planta com arquitetura na forma de 'taça', visando aumentar o número de frutos por planta.

Irrigou-se por gotejamento, composto de fitas gotejadoras com diâmetro de $16,4 \mathrm{~mm}$, vazão de $1,1 \mathrm{~L} / \mathrm{h}$ e com emissores espaçados em $40 \mathrm{~cm}$. O sistema de bombeamento foi composto por bomba centrífuga, com potência de $0,736 \mathrm{~kW}$, vazão de 0,5 a $11 \mathrm{~m}^{3} / \mathrm{h}$ e pressão manométrica de 23 a 31 mca. O sistema foi composto por filtros, manômetros, hidrômetros e registros. Foram instalados reguladores de pressão de $105 \mathrm{kPa}$ em cada uma das oito linhas de derivação.

As irrigações foram realizadas com turno de rega fixo de dois dias. A quantidade de água aplicada foi determinada com base na curva de retenção da água no solo e nas leituras do potencial mátrico $(\psi \mathrm{m})$ em tensiômetros com leitura digital. Em cada casa de vegetação foram instalados quatro tensiômetros nas profundidades de 20 e $40 \mathrm{~cm}$.

O sistema de fertirrigação operou independentemente dos sistemas de irrigação e de aplicação de $\mathrm{CO}_{2}$, sendo o reservatório da calda independente do reservatório de irrigação. A calda foi preparada em baldes de 20 litros e adicionada a um reservatório de 200 litros para posterior aplicação. A fertirrigação foi realizada de acordo com a análise química do solo (Tabela 1), conforme metodologia descrita anteriormente. A solução foi preparada no momento de cada fertirrigação, utilizando-se nitrato de potássio e nitrato de cálcio, na concentração de $17 \mathrm{~kg} /$ ha para ambos, baseado na análise química do solo.
Foram realizadas 12 aplicações com freqüência de 15 dias.

$\mathrm{O}$ sistema de aplicação de $\mathrm{CO}_{2}$ foi composto de um cilindro de $11 \mathrm{~kg}$, equipado com uma válvula reguladora de pressão, manômetro e fluxômetro com escala de 0,2 a 2,0 L/min para quantificar o volume de $\mathrm{CO}_{2}$ injetado via água de irrigação. A aplicação de $\mathrm{CO}_{2}$ ocorreu com freqüência de quatro dias. As doses de $\mathrm{CO}_{2}$ aplicadas foram quantificadas a cada aplicação por uma balança de precisão (legibilidade de $10 \mathrm{~g}$ ), sendo o cilindro pesado durante a aplicação. A utilização da balança foi devido ao fato de o fluxômetro não possibilitar rigoroso controle de $\mathrm{CO}_{2}$ injetado no sistema.

$\mathrm{O}$ tempo médio por aplicação de $\mathrm{CO}_{2}$ foi quatro, oito e doze minutos para as doses um, dois e três, respectivamente. Após esses tempos, aplicou-se apenas água para totalizar 30 minutos de irrigação. Esse procedimento foi utilizado para que o $\mathrm{CO}_{2}$ fosse eliminado do sistema entre aplicações sucessivas. Além disso, as aplicações foram iniciadas pela menor dose para minimizar a interferência entre um tratamento e outro. Após a aplicação do $\mathrm{CO}_{2}$, foi irrigado o tratamento sem $\mathrm{CO}_{2}$ (testemunha) com a mesma quantidade de água.

O ciclo produtivo da pimenta foi de 432 dias após o transplantio (DAT), tendo sido realizadas treze colheitas após os 127 DAT, no intervalo de sete dias entre uma colheita e outra. Após os 188 DAT foi realizada uma poda (04/04/07), devido ao estiolamento ocasionado pela baixa radiação no interior da casa-de-vegetação. Uma das estratégias para melhorar a radiação nesse ambiente foi a retirada da manta termorrefletora (aluminet, 50\%). A duração do ciclo antes da poda, considerando a época de semeadura, foi de 239 dias, e após a poda foi de 245 dias, totalizando 16 meses de cultivo.

As concentrações de macronutrientes (N, P, K, Ca, Mg e S) e de micronutrientes (B, $\mathrm{Cu}, \mathrm{Fe}, \mathrm{Mn}$ e $\mathrm{Zn}$ ) foram determinadas em dez folhas jovens dispostas na parte superior (terminal ou lateral) dos ramos, no final do ciclo antes da poda, correspondente ao final da colheita (187 dias após o transplantio). Após a poda, foi realizada a análise foliar no início da frutificação (404 dias após o transplantio) e no final do ciclo, correspondente à última colheita (484 dias após o transplante) com a mesma metodologia de coleta das folhas utilizada antes da poda. As análises foliares foram realizadas segundo a metodologia descrita por Malavolta (2006) no Laboratório de Ecologia Aplicada (ESALQ).

Para a avaliação estatística dos dados obtidos nas três épocas de amostragem, foi empregado o programa SAS (SAS, 1999); quando houve efeito significativo dos tratamentos, os dados foram submetidos à análise de regressão.

\section{RESULTADOS E DISCUSSÃO}

Verificou-se efeito linear significativo $(\operatorname{Pr}<0,05)$ para as doses de $\mathrm{CO}_{2}$ sobre a concentração de N, Fe, Mn e, para o $\mathrm{Zn}$ efeito quadrático significativo $(\operatorname{Pr}<0,05)$, aos 187 DAT (Figura 1). Com relação à concentração de $\mathrm{Ca}$, apresentou efeito linear significativo $(\operatorname{Pr}<0,01)$ das doses de $\mathrm{CO}_{2}$ sobre o teor de $\mathrm{Ca}$ nas folhas da pimenta aos 484 DAT (Figura 2). Para os demais macronutrientes e micronutrientes, não ocorreram efeitos significativos das doses de $\mathrm{CO}_{2}$ sobre a concentração nas folhas da pimenta nas diferentes datas de amostragens realizadas durante o ciclo da cultura.

As concentrações de macronutrientes $(\mathrm{g} / \mathrm{kg})$ nas folhas em ordem decrescente foram: N $(43,4), \mathrm{K}(25,5)$, $\mathrm{Ca}(16,9), \operatorname{Mg}(8,78), \mathrm{S}(2,41), \mathrm{P}(2,36)$, e a de micronutrientes $(\mathrm{mg} / \mathrm{kg})$ foram: $\mathrm{Cu}(596,1), \mathrm{Fe}(579,7), \mathrm{Mn}(147,2), \mathrm{B}$ $(61,6)$ e $\mathrm{Zn}(48,1)$, respectivamente. Segundo Pinto et al (2006), os macronutrientes mais absorvidos na cultura da pimenta são $\mathrm{N}(6,6), \mathrm{K}(6,4), \mathrm{Ca}(2,6)$, Mg (1,3), S (1,1) e P (0,7). Kano et al. (2013), avaliando a adição de $\mathrm{CO}_{2}$ e potássio na água de irrigação, não observaram efeito significativo no acúmulo de nutrientes na cultura do meloeiro, com exceção do potássio e do magnésio, com diminuição do acúmulo no final do ciclo em função da água carbonatada.

A concentração de $\mathrm{N}$ nas folhas, aos 187 DAT aumentou em função das doses de $\mathrm{CO}_{2}$, com valores de 49,3, 50,3, 53,7 e $59,5 \mathrm{~g} / \mathrm{kg}$ para T1, T2, T3 e T4, respectivamente. Branco et al. (2007) 


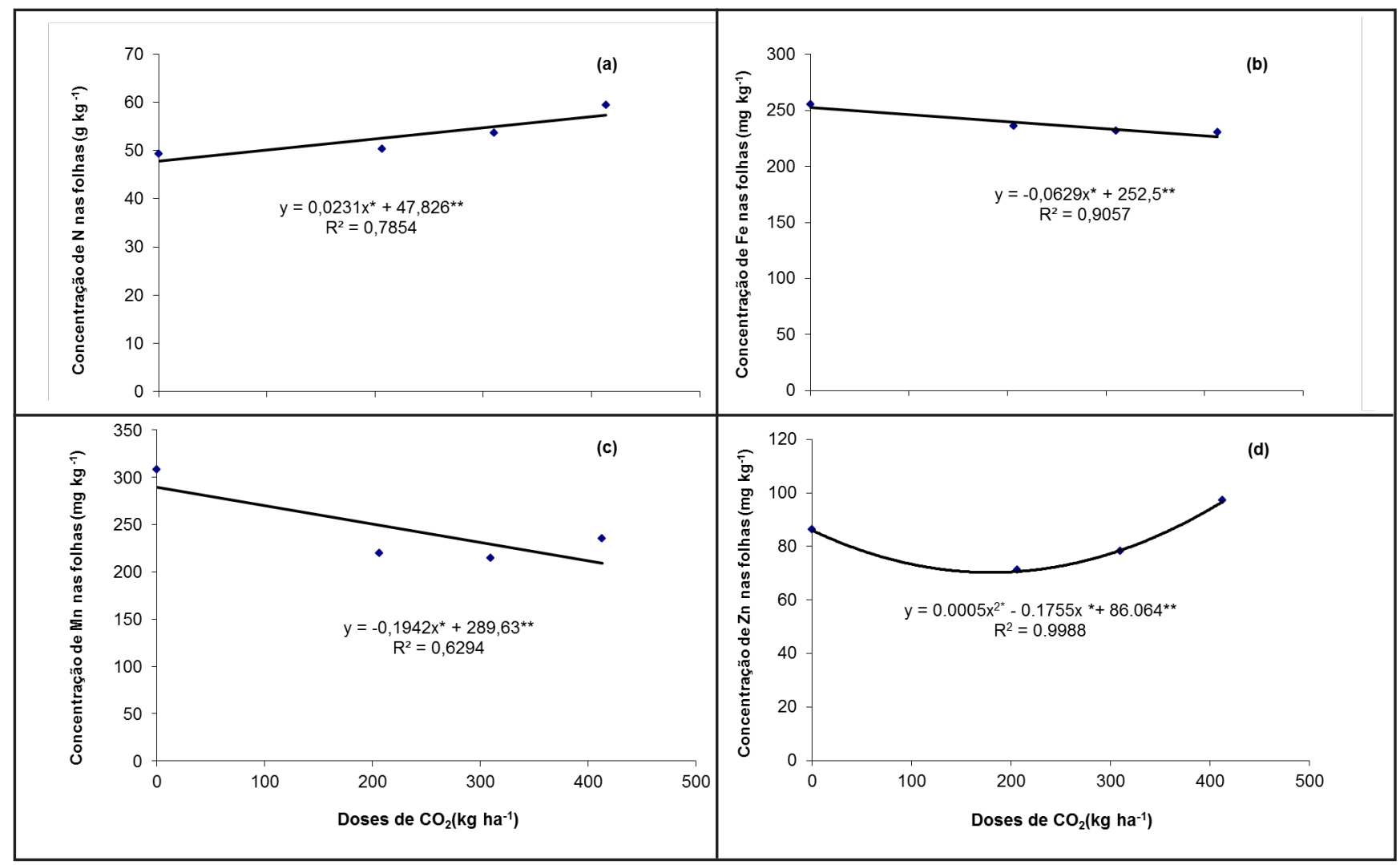

Figura 1. Concentração de nitrogênio (a), ferro (b), manganês (c) e zinco (d) nas folhas da pimenta 'Tabasco' aos 187 DAT em função das doses de $\mathrm{CO}_{2}$ aplicadas via irrigação por gotejamento \{nitrogen (a), iron (b), manganese (c) and zinc (d) content in the leaves of Capsicum frutescens at 187 DAT depending on the $\mathrm{CO}_{2}$ levels applied by drip irrigation $\}$. *** ${ }^{*}$ significativo pelo teste $\mathrm{t}$ ao nivel de 5 e $1 \%$, respectivamente (significant at 5 and 1\%, respectively, test t). Piracicaba, ESALQ, 2008.

Tabela 1. Resultados da análise química do solo utilizado no experimento (results of chemical analysis of the soil used in the experiment). Piracicaba, ESALQ, 2008.

\begin{tabular}{|c|c|c|c|c|c|c|c|c|c|c|c|c|c|c|c|c|c|}
\hline \multirow{3}{*}{ Amostra } & \multirow{3}{*}{$\begin{array}{c}\mathrm{pH} \\
\left(\mathrm{CaCl}_{2}\right)\end{array}$} & \multirow{3}{*}{$\begin{array}{c}\text { M.O. } \\
\left(\mathrm{g} / \mathrm{dm}^{3}\right)\end{array}$} & \multicolumn{9}{|c|}{ Macronutrientes } & \multirow{3}{*}{$(\%)$} & \multicolumn{5}{|c|}{ Micronutrientes } \\
\hline & & & $\mathbf{P}$ & $\mathbf{S}$ & $\mathbf{K}^{+}$ & $\mathrm{Ca}^{+2}$ & $\mathbf{M g}^{+2}$ & $\mathbf{A l}^{+}$ & $\mathbf{H}+\mathbf{A l}^{+}$ & SB & $\mathbf{T}$ & & $\mathbf{B}$ & $\mathrm{Cu}$ & $\mathrm{Fe}$ & Mn & $\mathbf{Z n}$ \\
\hline & & & \multicolumn{2}{|c|}{$\left(\mathrm{mg} / \mathrm{dm}^{3}\right)$} & \multicolumn{7}{|c|}{$\left(\mathrm{mmol} / \mathrm{dm}^{3}\right)$} & & \multicolumn{5}{|c|}{$\left(\mathrm{mg} / \mathrm{dm}^{3}\right)$} \\
\hline Estufa 1 & 5,0 & 10 & 10 & 25 & 1,9 & 25 & 15 & 0 & 22 & 41,2 & 63,2 & 65 & 0,28 & 1,5 & 26 & 14,8 & 1,4 \\
\hline Estufa 2 & 5,9 & 17 & 20 & 35 & 2,9 & 53 & 25 & 0 & 16 & 80,9 & 96,9 & 83 & 0,28 & 2,0 & 18 & 19,0 & 2,1 \\
\hline
\end{tabular}

aplicaram $\mathrm{CO}_{2}$ na dose de $12 \mathrm{~L} / \mathrm{min}$ na água de irrigação na cultura do tomate, e observaram que o $\mathrm{CO}_{2}$ não alterou o transporte de ${ }^{15} \mathrm{~N}$, mas resultou em aumento na produção de frutos comerciais por planta.

No caso da pimenta, a concentração de $\mathrm{N}$ nas folhas foi crescente, em função das doses de $\mathrm{CO}_{2}$ aplicadas através da água de irrigação, quando comparada à do tratamento sem $\mathrm{CO}_{2}$ (Figura 1a). Essa resposta é semelhante à encontrada por Kano et al (2013) que observaram incremento no conteúdo de $\mathrm{N}$ em folhas de meloeiro rendilhado, em função da irrigação carbonatada de $301,8 \mathrm{~kg} / \mathrm{ha}$ de $\mathrm{CO}_{2}$.

$\mathrm{O}$ incremento da absorção do nitrogênio pela pimenteira pode estar associado ao efeito do rebaixamento do $\mathrm{pH}$ do solo, em resposta à aplicação do $\mathrm{CO}_{2}$. Segundo Souza et al. (2010), o aumento da absorção de nutrientes pode estar associado à disponibilidade de certos nutrientes, que pode aumentar devido ao abaixamento do $\mathrm{pH}$ do solo, ocasionado pela aplicação do $\mathrm{CO}_{2}$ via água de irrigação; outro fator pode estar associado ao estimulo do crescimento de raízes ocasionado pelo $\mathrm{CO}_{2}$, na ri- zosfera, agindo de forma semelhante a um fitohormônio, num equilíbrio competitivo, com a concentração de etileno do ar no solo. Em função do aumento da concentração do N nas folhas com a utilização da aplicação de $\mathrm{CO}_{2}$ via água da irrigação, Paula et al. (2011) obtiveram incremento de $16 \%$ na dose de $451,95 \mathrm{~kg}$ $\mathrm{CO}_{2} /$ ha durante todo o ciclo da cultura da pimenta "Tabasco".

Quanto ao Fe, observou-se que a concentração na folha diminuiu, em função das doses de $\mathrm{CO}_{2}$ (Figura 1b); o mesmo comportamento foi observado, em relação ao $\mathrm{Mn}$, porém, com 


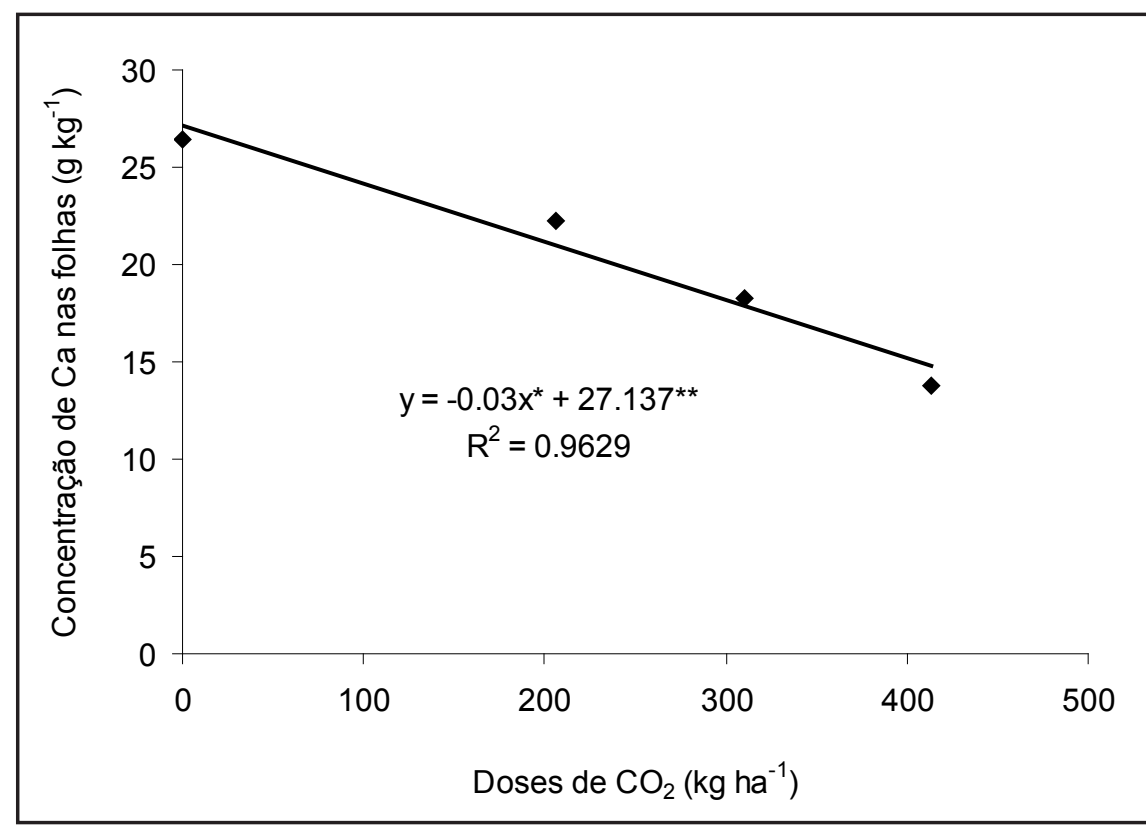

Figura 2. Concentração de Ca nas folhas da pimenta 'Tabasco' aos 484 DAT em função das doses de $\mathrm{CO}_{2}$ aplicadas via irrigação por gotejamento \{Ca concentration in leaves of Capsicum frutescens, on 484 DAT, depending on the $\mathrm{CO}_{2}$ levels applied by drip irrigation $\}$. **significativo pelo teste $\mathrm{t}$ ao nível de $1 \%$ de probabilidade; * significativo pelo teste $\mathrm{t}$ ao nível de 5\% de probabilidade. Piracicaba, ESALQ, 2008.

um aumento em $\mathrm{T} 4$, em relação a $\mathrm{T} 3$ e T2 (Figura 1c), aos 187 DAT. Essa redução na absorção se deveu ao fato de os teores de Fe e Mn estarem com sua concentração na folha próxima ao limite considerado máximo para a cultura que, segundo Trani (2007), a faixa de concentração de $\mathrm{Fe}$ nas folhas varia de 50 a $300 \mathrm{mg} / \mathrm{kg}$, enquanto a de $\mathrm{Mn}$ varia de 30 a $250 \mathrm{mg} / \mathrm{kg}$ na cultura do pimentão. Outra explicação deve-se ao fato desses nutrientes no solo estarem próximos do ponto de máxima concentração na solução do solo (Tabela 1) que corresponde a $20 \mathrm{mg} / \mathrm{L}$ para o manganês (Pereira et al., 2001).

A concentração de Zn (Figura 1d) diminuiu nos tratamentos T2 e T3, em relação a $T 1$, o que não foi observado em T4, no qual a concentração de Zn foi maior, quando comparada à do tratamento sem $\mathrm{CO}_{2}$ (T1), resultado esse semelhante ao obtido por Cararo \& Duarte (2002) e Kano et al. (2013) para as culturas do tomate e meloeiro, respectivamente. Kano et al (2013), avaliando a adição de agua carbonatada na cultura do meloeiro, observaram que os teores de macronutrientes tiveram um incremento com o uso desta tecnologia, e para os micronutrientes como $\mathrm{Zn}$ e
Mn obtiveram aumento no acúmulo nas folhas.

A concentração de $\mathrm{Ca}$ nas folhas apresentou uma resposta linear decrescente, em função do aumento das doses de $\mathrm{CO}_{2}$ (Figura 2), semelhante ao encontrado por Kano et al. (2013), que observaram menor concentração de $\mathrm{Ca}$ em melão rendilhado com a utilização de água carbonatada. $\mathrm{OCO}_{2}$ aplicado via irrigação promove a redução momentânea do $\mathrm{pH}$ do solo e o maior crescimento das raízes, aumentando a disponibilidade de nutrientes (Pinto et al., 2000; Araújo \& Botrel, 2010). De acordo com Trani et al. (2007), os valores adequados de $\mathrm{Ca}$ variam entre 15 e $35 \mathrm{~g} / \mathrm{kg}$, estando os valores observados neste trabalho de acordo com a faixa ideal, com exceção de T4. Apesar de T4 apresentar valor abaixo do ideal, não foram observados sintomas visuais de podridão apical dos frutos, decorrentes da deficiência de $\mathrm{Ca}$, aos 484 DAT (Figura 2).

Conclui-se que o $\mathrm{CO}_{2}$ teve efeito significativo nos teores de N, Fe, Mn e $\mathrm{Zn}$ aos 187 DAT e no Ca aos 484 DAT. O $\mathrm{CO}_{2}$ ocasionou aumento na concentração de $\mathrm{N}$ e $\mathrm{Zn}$ nas folhas de pimenta Tabasco e um decréscimo na concentração de $\mathrm{Fe}$, $\mathrm{Mn}$ e Ca. Os valores observados dos nutrientes nas folhas foram suficientes, sem causar desiquilíbrio nutricional, não ocorrendo falta e nem excesso dos mesmos.

\section{AGRADECIMENTOS}

Os autores agradecem ao Ministério da Ciência e Tecnologia (MCT), ao Conselho Nacional de Desenvolvimento Científico e Tecnológico (CNPq) e à Fundação de Amparo à Pesquisa do Estado de São Paulo (FAPESP), pelo apoio financeiro a esta pesquisa, através do Instituto Nacional de Ciência e Tecnologia em Engenharia da Irrigação (INCT-EI).

\section{REFERÊNCIAS}

ARAÚJO WF; BOTREL TA. 2010. Influência do $\mathrm{CO}_{2}$ na água de irrigação e da cobertura plástica no solo na abobrinha. Revista Ciência Agronômica 41: 216-221.

BEZERRA NETO F; BARROS JUNIOR AP; AROUCHA EMM; OLIVEIRA EQ; SILVA EO. 2008. Conteúdo de nutrientes na folha de alface em sistema consorciado com cenoura sob diferentes densidades populacionais. Caatinga 21: 116-123.

BRANCO RBF; GOTO R; CARNEIRO JUNIOR AG; GUIMARÃES VF; RODRIGUES JD; TRIVELIN PCO; SILVEIRA LV. 2007. Enxertia e água de irrigação carbonatada no transporte de ${ }^{15} \mathrm{~N}$ e na produção do tomateiro. Revista Brasileira Engenharia Agrícola e Ambiental 11: 374-379.

CAMARGO OA; MONIZ AC; JORGE JA; VALADARES JMAS. 2009. Métodos e análise química mineralógica e física dos solos do Instituto Agronômico de Campinas. Boletim Técnico, 106. Campinas, 72p.

CARARO DC; DUARTE SM. 2002. Injeção de $\mathrm{CO}_{2}$ e lâminas de irrigação em tomateiro sob estufa. Horticultura Brasileira 20: 432-437.

D'ALBUQUERQUE JUNIOR BSD; FIZZONE JA; DUARTE SN; MINGOTI R; DIAS NS; SOUSA VF. 2007. Qualidade física e química de frutos de meloeiro rendimento cultivado sob diferentes épocas de aplicação de $\mathrm{CO}_{2}$ via água de irrigação. Irriga 12: 273-280.

EMBRAPA. 2006. Sistema de produção de pimentas (Capsicum $\mathrm{sp}$ ). Disponível em: $<$ http://www.cnph.embrapa.br/capsicum/ cultivo.htm>. Acesso em: 18 janeiro 2013.

EPAMIG. 2006. Cultivo da pimenta. Informe agropecuário 27: 7-15.

FRIZZONE JA; CARDOSO SS; REZENDE R. 2005a. Produtividade e qualidade de frutos de meloeiro cultivado em ambiente protegido com aplicação de dióxido de carbono e de potássio via água de irrigação. Acta Scientiarum Agronomy 27: 707-717. 
FRIZZONE JA; D’ALBUQUERQUE JUNIOR BS; REZENDE R. 2005b. Aplicação de dióxido de carbono via água de irrigação em diferentes fases fenológicas da cultura do meloeiro cultivado em ambiente protegido. Acta Scientiarum Agronomy 27: 667-675.

FURLAN RA; REZENDE FC; ALVES DRB; FOLEGATTI MV. 2002. Lâmina de irrigação e aplicação de $\mathrm{CO}_{2}$ na produção de pimentão cv. Mayata, em ambiente protegido. Horticultura Brasileira 20: 547-550.

KANO C; CARMELLO QAC; FRIZZONE JA; CARDOSO SS. 2013. Teor e acúmulo de nutrientes pelo meloeiro rendilhado cultivado com potássio e $\mathrm{CO}_{2}$ na água de irrigação. Biotemas 26: 19-28.

KHAN MUH; CHTTHA TH; SALEEN N. 2005. Influence of different irrigation intervals on growth and yield of bell pepper (Capsicum annum). Journal Agriculture and Biological Science 1: 125-128.

MALAVOLTA E. 2006. Manual de nutrição mineral de plantas. São Paulo. Editora Agronômica Ceres, 638p.

NOVAIS RF; ALVAREZ VH; BARROS NF; FONTES RLF; CANTARUTTI RB; NEVES
JCL 2007. Fertilidade do solo. Viçosa: Sociedade Brasileira de Ciência de Solo, $1017 \mathrm{p}$.

PAULA FLM; FRIZZONE JA; PAULA AL; DIAS CTS; SOARES TM. 2011. Produção da pimenta Tabasco com aplicação de $\mathrm{CO}_{2}$, utilizando-se irrigação por gotejamento. Acta Scientiarum 33: 133-138.

PEREIRA, G.D.; BERTONI, J.C.; CARVALHO, J.G.; MORAIS, A.R. 2001. Doses e modos de adubação com manganês e seus efeitos na produção da cultura do arroz. Revista Brasileira de Ciência do Solo 25: 625-633.

PINTO CMF; PUIATTI M; CALIMAN FRB; MOREIRA GR; MATTOS RN. 2006. Clima, época de semeadura, produção de mudas, plantio e espaçamento na cultura da pimenta. Informe Agropecuário 27: 40-49.

PINTO JM; BOTREL TA; MACHADO EC. 2000. Uso do dióxido de carbono na agricultura: revisão bibliográfica. Ciência Rural 30: 919-925.

PINTO JM; BOTREL TA; MACHADO EC; FEITOSA FILHO JC. 2001. Aplicação de $\mathrm{CO}_{2}$ via água de irrigação em relação à produtividade do meloeiro. Scientia Agricola
58: 33-38.

PORTZA; MARTINS CAC; LIMA E; ZONTAE. 2006. Teores e acúmulo de nutrientes durante o ciclo da mandioquinha-salsa em função da aplicação de Nitrogênio, Fósforo e Potássio. Horticultura Brasileira 24: 329-333.

RAIJ B; CANTARELLA H; QUAGGIO JA; FURLANI AMC. 1997. Recomendações de adubação e calagem para o Estado de São Paulo. 2. ed. rev. atual. Campinas: IAC. (Boletim técnico, 100). 285p.

SAS INSTITUTE. 1999. SAS: User's guide statistics: Version 8.0 edition. Cary. 956p.

SOUZA LH; NOVAIS RF; ALVAREZ VH; VILLANI EMA. 2010. Efeito do pH do solo rizosférico e não rizosférico de plantas de soja inoculadas com Bradyrhizobium japonicum na absorção de boro, cobre, ferro, manganês e zinco. Revista Brasileira de Ciência do Solo 34: 1641-1652.

TRANI PE. 2007. Calagem e adubação para hortaliças sob cultivo protegido. Disponível em: http://www.infobibos.com/ artigos/2007_1/cp/index.htm/. Acesso em: 5 maio de 2008 . 